\title{
Luciola hypertelescope space observatory: versatile, upgradable high-resolution imaging, from stars to deep-field cosmology
}

\author{
Antoine Labeyrie · Hervé Le Coroller • Julien Dejonghe • \\ Olivier Lardière • Claude Aime • Kjetil Dohlen • Denis Mourard • \\ Richard Lyon • Kenneth G. Carpenter
}

Received: 9 January 2008 / Accepted: 12 August 2008 / Published online: 17 September 2008 (c) Springer Science + Business Media B.V. 2008

\begin{abstract}
Luciola is a large $(1 \mathrm{~km})$ "multi-aperture densified-pupil imaging interferometer", or "hypertelescope" employing many small apertures, rather than a few large ones, for obtaining direct snapshot images with a high information content. A diluted collector mirror, deployed in space as a flotilla of small mirrors, focuses a sky image which is exploited by several beam-
\end{abstract}

\footnotetext{
A proposal to the European Space Agency for the Cosmic Vision 2015-2025 plan.

Refereed, condensed and updated version of the original proposal (available for reference at www.oamp.fr/infoglueDeliverLive/www/OHP/Actualit\%E9s? contentId =1148).
}

\footnotetext{
A. Labeyrie $(\otimes)$

Collège de France, Paris, France

e-mail: antoine.labeyrie@obs-azur.fr
}

A. Labeyrie $\cdot$ D. Mourard
Observatoire de la Côte d'Azur, Caussols, France

H. Le Coroller

Observatoire de Haute Provence, Saint Michel l'Observatoire, France

J. Dejonghe

Collège de France, Saint Michel l'Observatoire, France

O. Lardière

Adaptive Optics Lab, University of Victoria, Greater Victoria, BC, Canada

C. Aime

Université de Nice-Sophia Antipolis, Nice, France

K. Dohlen

Observatoire Astronomique Marseille Provence, Marseille, France

R. Lyon · K. G. Carpenter

NASA Goddard Space Flight Center, Greenbelt, MD, USA 
combiner spaceships. Each contains a "pupil densifier" micro-lens array to avoid the diffractive spread and image attenuation caused by the small subapertures. The elucidation of hypertelescope imaging properties during the last decade has shown that many small apertures tend to be far more efficient, regarding the science yield, than a few large ones providing a comparable collecting area. For similar underlying physical reasons, radio-astronomy has also evolved in the direction of many-antenna systems such as the proposed Low Frequency Array having "hundreds of thousands of individual receivers". With its high limiting magnitude, reaching the $m_{\mathrm{v}}=30$ limit of HST when 100 collectors of $25 \mathrm{~cm}$ will match its collecting area, high-resolution direct imaging in multiple channels, broad spectral coverage from the 1,200 $\AA$ ultra-violet to the $20 \mu \mathrm{m}$ infra-red, apodization, coronagraphic and spectroscopic capabilities, the proposed hypertelescope observatory addresses very broad and innovative science covering different areas of ESA's Cosmic Vision program. In the initial phase, a focal spacecraft covering the UV to near IR spectral range of EMCCD photon-counting cameras (currently 200 to 1,000 nm), will image details on the surface of many stars, as well as their environment, including multiple stars and clusters. Spectra will be obtained for each resel. It will also image neutron star, black-hole and micro-quasar candidates, as well as active galactic nuclei, quasars, gravitational lenses, and other Cosmic Vision targets observable with the initial modest crowding limit. With subsequent upgrade missions, the spectral coverage can be extended from $120 \mathrm{~nm}$ to $20 \mu \mathrm{m}$, using four detectors carried by two to four focal spacecraft. The number of collector mirrors in the flotilla can also be increased from 12 to 100 and possibly 1,000. The imaging and spectroscopy of habitable exoplanets in the mid infra-red then becomes feasible once the collecting area reaches $6 \mathrm{~m}^{2}$, using a specialized mid infra-red focal spacecraft. Calculations (Boccaletti et al., Icarus 145:628-636, 2000) have shown that hypertelescope coronagraphy has unequalled sensitivity for detecting, at mid infra-red wavelengths, faint exoplanets within the exo-zodiacal glare. Later upgrades will enable the more difficult imaging and spectroscopy of these faint objects at visible wavelengths, using refined techniques of adaptive coronagraphy (Labeyrie and Le Coroller 2004). Together, the infra-red and visible spectral data carry rich information on the possible presence of life. The close environment of the central blackhole in the Milky Way will be imageable with unprecedented detail in the near infra-red. Cosmological imaging of remote galaxies at the limit of the known universe is also expected, from the ultra-violet to the near infra-red, following the first upgrade, and with greatly increasing sensitivity through successive upgrades. These areas will indeed greatly benefit from the upgrades, in terms of dynamic range, limiting complexity of the objects to be imaged, size of the elementary "Direct Imaging Field", and limiting magnitude, approaching that of an 8-m space telescope when 1,000 apertures of $25 \mathrm{~cm}$ are installed. Similar gains will occur for addressing fundamental problems in physics and cosmology, particularly when observing neutron stars and black holes, single or binary, including the giant black holes, with accretion disks and jets, in active galactic nuclei beyond the Milky Way. Gravitational lensing and micro- 
lensing patterns, including time-variable patterns and perhaps millisecond lensing flashes which may be beamed by diffraction from sub-stellar masses at sub-parsec distances (Labeyrie, Astron Astrophys 284:689, 1994), will also be observable initially in the favourable cases, and upgrades will greatly improve the number of observable objects. The observability of gravitational waves emitted by binary lensing masses, in the form of modulated lensing patterns, is a debated issue (Ragazzoni et al., MNRAS 345:100-110, 2003) but will also become addressable observationally. The technology readiness of Luciola approaches levels where low-orbit testing and stepwise implementation will become feasible in the 2015-2025 time frame. For the following decades beyond 2020, once accurate formation flying techniques will be mastered, much larger hypertelescopes such as the proposed $100 \mathrm{~km}$ Exo-Earth Imager and the 100,000 km Neutron Star Imager should also become feasible. Luciola is therefore also seen as a precursor toward such very powerful instruments.

Keywords Space interferometer $\cdot$ Hypertelescope $\cdot$ High-resolution • Exo-planet

\section{Introduction}

Following the initial operation of a terrestrial "two-telescope interferometer" by some of us in 1974, today's largest existing telescopes became involved in interferometric observations with baselines reaching several hundred meters. Their science yield is increasingly rich, even though the atmospheric turbulence markedly degrades their theoretical performance. There is general agreement that space versions of such instruments will gain enormously from the perfect "seeing" above the atmosphere. This prompted the early TRIO [2,3], and SAMSI [4] proposals for space interferometers with formation flying elements. More than a decade later the idea began attracting considerable support and study efforts, initially by ESA for DARWIN [7], then also by NASA for its Terrestrial Planets Finder (TPF) as they moved from "solid" to formation-flying versions of these infra-red interferometers.

Following the theoretical description of "hypertelescopes" [8], a class of direct-imaging interferometer using multiple apertures and a "densified pupil", terrestrial and space versions of such instruments were proposed [10, 14, 15]. As explained in the book of Labeyrie et al. [1], they typically use a multiaperture Fizeau interferometer equipped with a small image-relay stage at the focal plane, containing an array of tiny inverted Galilean telescopes which magnify each sub-pupil to concentrate their diffracted light and intensify the interference pattern (Fig. 1).The image-forming capability of Fizeau interferometers is preserved, although it becomes confined within a limited field of view, the "Direct Imaging Field" (DIF). Its sky diameter is $\lambda / s$, if $\lambda$ is the wavelength and s the sub-aperture spacing, when the pupil is fully densified, i.e. when the sub-pupils fill the pupil [1]. The direct image of a compact object fitting within the DIF is intensified, up to a million times with the initial 


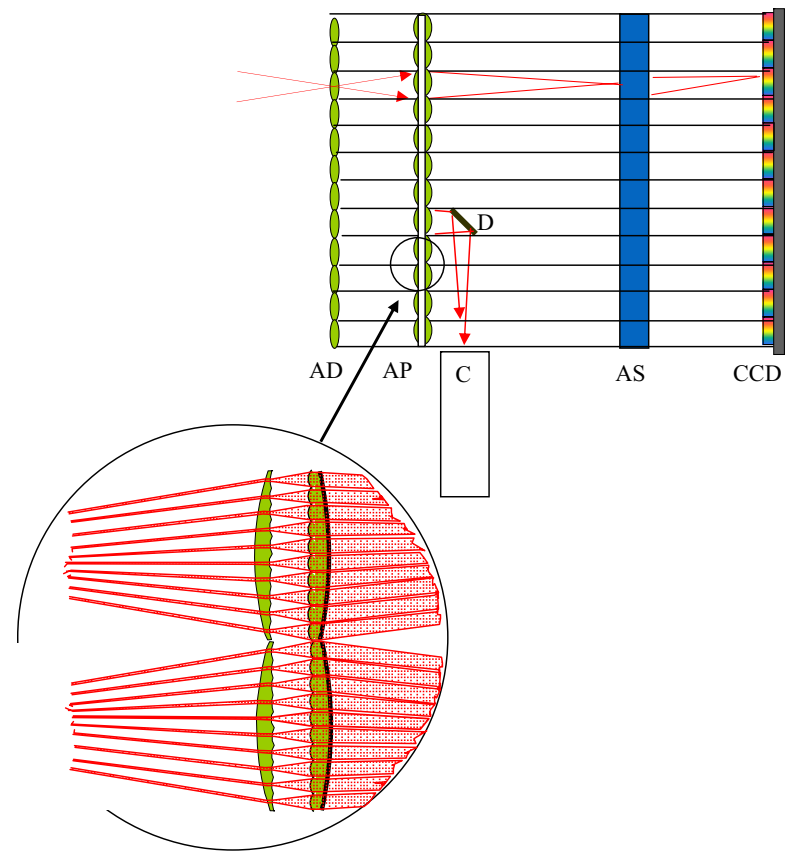

Fig. 1 Beam-combineroptics, with adjacent spectro-imaging channels, having separate pupil densifiers: $A D$ primary Fizeau focal plane with arrayed field lenses, each sized for matching the diffractive envelope and feeding a separate imaging channel; $A P$ array of micro-scale pupil densifiers in the relayed pupils; $A S$ array of faceted field-gratings in the relayed images; $C C D$ common detector for all field channels, recording in each a spectrum of each resel. The AP densifier array (magnified detail below) has two plano-convex lens arrays, the facing flat sides of which carry smaller curved facets. These are respectively concave and convex, each pair of facing facets behaving as a tiny Galilean telescope which magnifies the sub-pupils. The tens of narrow "Direct Imaging Fields" (DIF) simultaneously spectro-imaged are separated by gaps on the sky, but they can be filled, for stitching a continuous image, with a sequence of exposures having slightly offset pointing. Correction for local field aberrations is also included in the entrance face of each pupil densifier. In upgraded versions, adjustable deflecting wedges (not shown), at the entrance of the pupil densifier array AP, can independently center each DIF onto an object of interest. One of the imaging channels feeds a coronagraph $\mathrm{C}$ for exoplanet imaging

12-aperture Luciola. For a broader instantaneous sky coverage by a focal spaceship, many DIFs can be exploited in parallel with an array of micro-scale pupil densification channels (Fig. 1).

For all interferometers, including Fizeau and hypertelescopes having $\mathrm{N}$ apertures, a common limitation is "field crowding": the contrast or visibility of the interference pattern becomes vanishingly small if more than $\mathrm{N}^{2}$ point sources are present within the "diffractive envelope" or "sky lobe" of the sub-apertures. On extended clusters of point sources, the resulting maximal number of such sources, or "active resels", tolerable per square arc second is:

$$
s_{\mathrm{as}}=1.410^{-9} \pi^{3} N^{2} d^{2} \lambda^{-2=} 1.410^{-9} \pi^{-3} A^{2} d^{-2} \lambda^{-2}
$$


where $d$ is the diameter of the sub-apertures and $A=N d^{2}$ is the collecting area [31, 32]. With $N=1,000$ apertures of $25 \mathrm{~cm}$, the crowding limit reaches 5 million point sources per square-arc second at visible wavelengths. This is suitable for directly imaging complex objects such as deep cosmological fields containing many small galaxies. The crowding limit further improves if the aperture pattern is varied or rotated during the exposure, as illustrated in Figs. 2 and 3, but it is degraded in the presence of photon noise, or thermal noise in the infra-red.

With a given collecting area A, smaller and more numerous sub-apertures improve greatly the crowding limit. Interestingly, it does not depend on the global array size D: enlarging a given array by spreading apart its apertures thus improves the resolution without degrading the crowding limit, expressed as the limiting number of "active resels" per sky area. However, in a given observed field, improving the resolution tends to create more "active resels" as multiple stars or galaxies become resolved, and this can limit the useful array span if no apertures are added.

The hypertelescope optics also provides a large gain in imaging performance with respect to aperture-synthesis interferometers having a comparable resolution and collecting area, but in the form of few apertures. The gain in
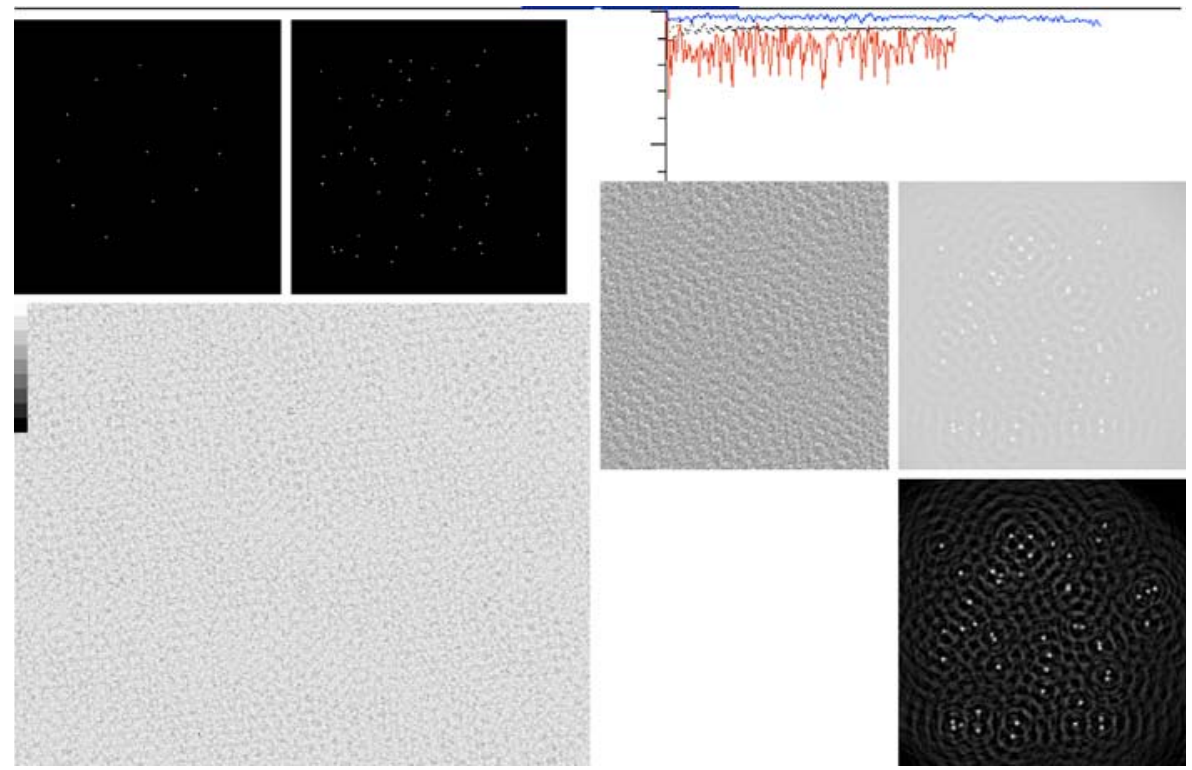

Fig. 2 Numerical simulation of Fizeau imaging with 12 sub-apertures (top left), on a single star (bottom left, in log scale with decade calibration) and on a cluster of 50 stars (object at top center, images at bottom right). The three images are plotted in linear scale and made respectively (clockwise) with a fixed aperture, with a rotated aperture, and with a rotated aperture plus subtraction of a uniform background. Half-profiles are plotted at top right in log intensities (marks indicate decades) for the spread function (lower curve), its angular average (median curve) and the fixed-aperture image (upper curve) 

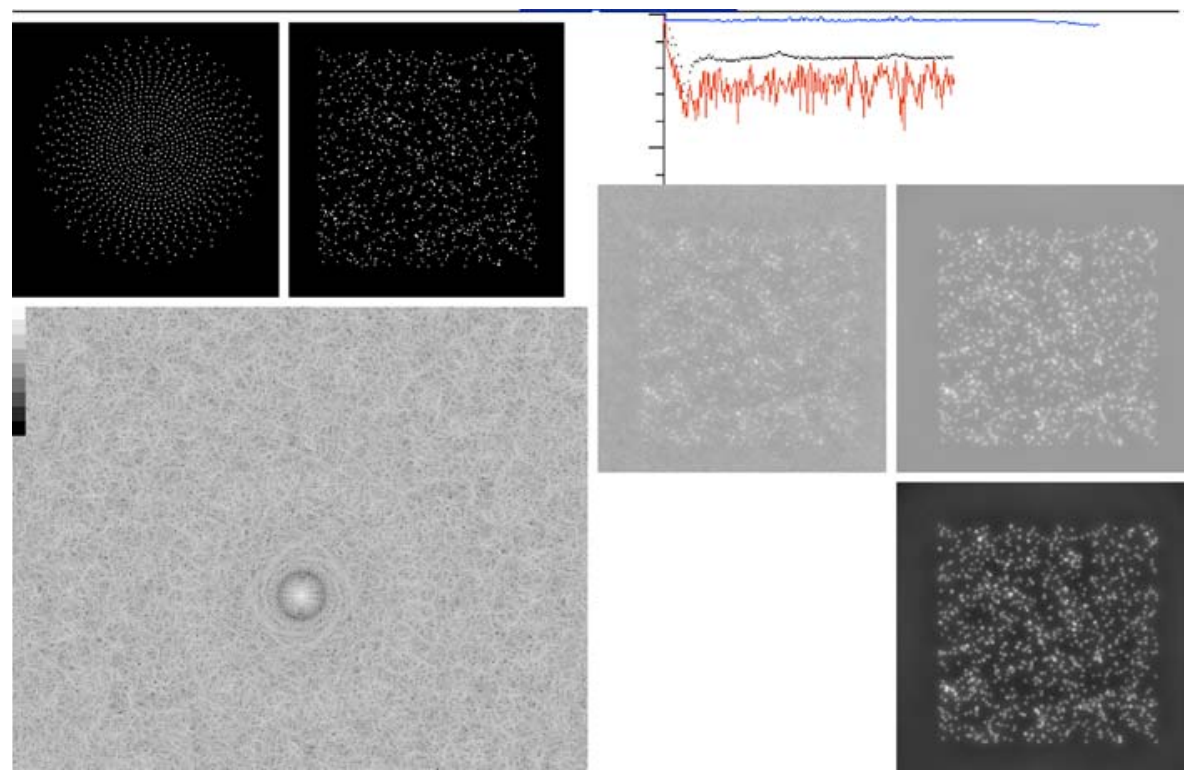

Fig. 3 Improved imaging with 1,000 apertures and 1,000 stars, displayed like in Fig. 2. The aperture pattern is an Archimedian spiral, with sub-aperture spacing increased along the arm for apodization. The sidelobes in the spread function are markedly attenuated with the numerous apertures, and the direct image accurately reproduces the star cluster details

signal/noise ratio, resulting from the improved dynamic range in direct images obtained with more apertures, is indeed of the order of $\mathrm{N}^{7 / 4}$, amounting to 8,000 or $10^{5}$ respectively if the number of collector mirrors reaches 100 or $1,000[31]$.

It is therefore of advantage to exploit many small apertures, in hypertelescope fashion, rather than a few large ones. Not only is the science yield greatly improved, with the larger DIF, increased dynamic range and higher crowding limit, but some operational aspects are also facilitated and the mirror cost reduced, as discussed in Section 2.11. Snapshot images, rich in information content, are directly recorded by the focal camera. Dispersive elements can also exploit the spectroscopic information contained in each resel. Such combined image and spectroscopic data is highly valuable for constraining astrophysical models of varied objects. With respect to the modelfitting efforts heretofore made with tentative image reconstructions from few apertures, the better images from numerous apertures give more significant science results, as demonstrated in radio-interferometry since the construction of the Very Large Array.

The limiting magnitude for detecting a point source against the sky background, using a photon-limited camera, is in principle the same for a hypertelescope, having sub-apertures of any size, as for a monolithic telescope of identical collecting area. This can be established by considering a many- 
aperture version of Michelson's 20 or 50-ft interferometer architectures, which may be seen as embryonic hypertelescopes: varying the spacing of the entrance mirrors leaves invariant the image of a non-resolved star amidst the sky background halo, itself also invariant. Densifying the entrance aperture, by moving its mirrors inward all the way to a compact aperture, thus preserves the signal/(photon noise) ratio of the star image.

The limiting magnitude of Luciola can be calculated with the expressions given for the EED proposal by Boccaletti et al. [16] \& Riaud et al. [20], and for the Epicurus proposal [17]. It is expected to reach $m_{\mathrm{v}}=32$ with the $63 \mathrm{~m}^{2}$ collecting area offered by 1,000 mirrors of $25 \mathrm{~cm}$.

A terrestrial hypertelescope prototype, very similar in its optical design to the Luciola but much smaller, is under development following "first light" when fringes were obtained on Vega with a balloon-suspended camera [24]. In space, the efficiency of such optical architectures which are diluted versions of the Arecibo radio-telescope is further increased since many focal combiners can be utilized to better exploit the broad sky image formed along the focal surface of the primary spherical collector. The aperture size is also virtually unlimited in space, like the number of sub-apertures.

The Luciola hypertelescope is conceived as an observatory with broad science capabilities, from exoplanets to stellar physics and deep-field cosmology. The initial configuration, with twelve or more small apertures, is expected to evolve through successive upgrades toward a large and powerful hypertelescope having hundreds of apertures, expected to contribute eminently to astrophysics in the coming decades at ultra-violet, visible, and infra-red wavelengths toward $20 \mu \mathrm{m}$.

With respect to the previous proposals for space hypertelescopes made by some of us, i.e. the Exo-Earth Discoverer submitted to NASA in 1998 as a candidate version of TPF-I [12, 21], and the "bare-bone" Epicurus submitted to ESA [17], the science scope of Luciola (Fig. 4) is greatly extended, particularly toward the direct imaging of cosmological deep fields, by using smaller apertures and progressively increasing their number to hundreds. 1,000 mirrors of $0.25 \mathrm{~m}$ are indeed equivalent in collecting area to an $8 \mathrm{~m}$ telescope, and reach the same high limiting magnitude. Luciola also covers a broader spectral range, from the deep ultra-violet to the mid infra-red. And we further developed the attractive option of using solar propulsion for the nano-satellites which carry the collector mirrors. A simplified solution also emerged for accurate laser metrology, allowing piston phasing on even the faintest sources (Fig. 7).

Space brings extraordinary possibilities for hypertelescopes, eventually up to the $100,000 \mathrm{~km}$ "Neutron Star Imager" versions capable of angularly resolving the $20 \mathrm{~km}$ neutron star of the Crab Pulsar. Before that, a $100 \mathrm{~km}$ ExoEarth Imager (EEI) having 100 mirrors of $3 \mathrm{~m}$, or 1,000 of $1 \mathrm{~m}$, is expected to show details of an exo-Earth with sufficient resolution and spectral information to analyze any green spots in terms of photosynthetic life [25].

But preliminary steps are obviously needed. For a first-generation space hypertelescope, the present proposal attempts to strike an optimal balance 


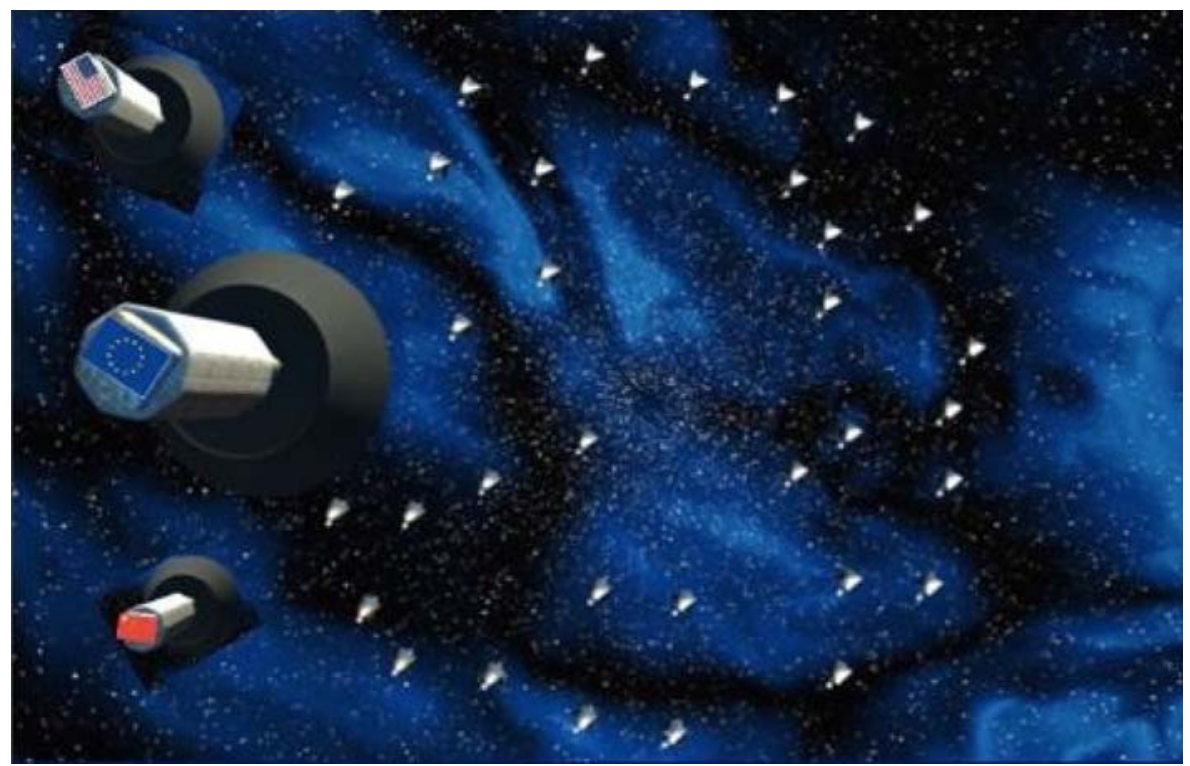

Fig. 4 Artist view of the Luciola flotilla with its formation flight of many small collector mirrors, operating like a giant diluted mirror. Focal beam-combiner spacecraft, in the foreground, independently exploit the sky image formed at the focal surface. Some of them can be built by space agencies other than ESA, and delivered with upgrade missions. Tens of combiners, and hundreds of small collector spacecraft, can be progressively incorporated for greatly expanded science. The metrology spacecraft, emitting laser beams from the curvature center of the collector locus, is in the back of the observer and not visible

between science, cost and reliability by using a modular architecture, enriched through planned upgrade missions.

The proposed Luciola hypertelescope is a general-purpose high-resolution observatory designed for different forms of observing, with its spectro-imaging and coronagraphic attachments. Planned upgrade missions will bring additional aperture elements, in the form of mirror segments carried by nanosatellites, and additional instruments may also later be added, in the form of dedicated focal spacecraft. The collector array will then be reconfigured from the initial paraboloid shape to a spherical shape (a deformation amounting to less than $10 \mathrm{~mm}$ for the $1 \mathrm{~km}$ flotilla), so that several, and possibly many, such focal spacecraft can simultaneously observe different targets in the broad collector field, a sizeable gain in terms of science throughput.

\section{Scientific objectives}

The efficient direct imaging with high angular resolution and high limiting magnitude, exceeding that of HST when 100 mirrors of $25 \mathrm{~cm}$ will be used, the broad wavelength range and image spectroscopy all result in highly diversified 
science objectives, covering several areas in the Cosmic Vision programme of ESA. The observable wavelength range can in principle be comparable to that exploited by HST, from the Lyman alpha ultra-violet $(1,200 \AA)$ to the nearinfra-red. It will be extended toward the mid infra-red since the solar thruster of each collector nano-satellite also serves as a sunshield, resembling that of the JWST. With a similar multi-layer insulation backing the sunshield, the stellar mirror located in its shadow is passively cooled, sufficiently for observing the mid infra-red to $20 \mu \mathrm{m}$. This is of interest for imaging habitable exoplanets with a specialized mid infra-red focal spacecraft containing a coronagraph and actively cooled detector. The ultra-violet limit is mostly defined by the coating types available for the collector mirror elements, typically aluminum with a protective magnesium fluoride coating like in HST.

In the following, science goals mentioned in ESA's Cosmic Vision science selection are indicated in italics.

\subsection{From gas and dust to stars and planets}

"Map the birth of stars and planets by peering into the highly obscured cocoons where they form"

With the passively cooled collector mirrors and infra-red optics in a dedicated infra-red focal spacecrfat, the infra-red range extending toward $20 \mu \mathrm{m}$ with 1 milliarcsecond resolution provides interesting capabilities in the way of penetrating dust regions and detecting protoplanetary features. On these rather extended objects, the fundamental crowding limitation of all interferometers, which improves as $\mathrm{N}^{2}$, gives a large advantage for the Luciola as it will be up-graded to hundreds of apertures.

\subsection{From exoplanets to biomarkers}

"Search for planets around stars other than the Sun, looking for biomarkers in their atmospheres, and image them"

Luciola has coronagraphic channels for producing images and lowresolution spectra of habitable planets near their parent star. In the 10-20 $\mu \mathrm{m}$ infra-red, habitable planets having $10^{-6}$ relative luminosity with respect to the parent star are imageable. As calculated by Boccaletti et al. [16], Luciola gains sensitivity for their detection against the exo-zodiacal nebula, if compared at equal collecting area with uni-axial nulling interferometers such as the DARWIN and TPF-I.

In the visible, a hot Jupiter such as 51 Pegasi b, only 10,000 times fainter than its parent star and spaced by 3.4 arc millisecond, is imageable with the baselines considered. Even its angular diameter, estimated at 70 micro arc seconds, becomes resolved in the near ultra-violet. Epsilon Eridani b, the closest known exoplanet, at 3.3 parsecs, is also resolved, and imageable with $12 \times 12$ resels in the Lyman alpha ultra-violet, if sufficiently bright in this range. Such resolution can suffice to detect morphology details such as bands, a red 
dot, and perhaps aurora patterns, and to analyze them with low-resolution spectroscopy. Exoplanet transits, now detected by even amateur photometry on many stars, will also be imageable in the form of a dark spot, more or less resolved, crossing the well resolved disk of the parent star. The absorption lines in the planet's outer atmosphere are then detectable with increased sensitivity, in comparison with unresolved spectroscopy.

Habitable and Earth-like planets are notoriously more difficult to image at visible wavelengths, where their contrast is $10^{-9}$ or even $10^{-10}$. It may however become possible with Luciola by using the extreme coronagraphy techniques [34] developed in the recent years (Section 5). Low-resolution spectra, then provided by the spectrographic attachment, provide indirect bio-markers in the form of absorption lines by biogenic molecules, which complement those obtainable at infra-red wavelengths. The direct spectral features of photosynthetic absorption by "exo-chlorophyll" are more difficult to detect on a planet, unless it is thickly vegetated, pending resolved images with larger instruments such as the Exo-Earth Imager.

Other by-products of exoplanet coronagraphy will be images, and spectroscopic data on exo-zodiacal clouds and debris disks, coronal emissions, circumstellar disks and jets.

\subsection{What are the fundamental physical laws of the universe?}

Gravitational waves It has been suggested [9] that the patterns of gravitational micro-lensing, formed when a mass is on the line-of-sight to a remote background compact source, may be modulated, in intensity or position, if the lensing mass is also a source of gravitational radiation. Damour and EspositoFarnese [9], and Kopeikin and Korobkov [22] have calculated that no such effects are observable in the framework of General Relativity, but Ragazzoni et al. [23] find instead that they may be observable. Bracco and Teyssandier [13] also find that such effects may be consistent with scalar-tensor theories of gravity. In this respect, it is of interest to observe short-period binary stars, possible sources of gravitational radiation, together with background sources. Detecting modulated lensing patterns in their close vicinity would help discriminating these theories, while also perhaps directly evidencing the emission of gravitational radiation. Slower binary stars also produce modulated microlensing patterns which are worth observing, although not in the radiative regime, as discussed by Dubath et al. [29].

\subsection{Matter under extreme conditions}

"Probe gravity theory in the very strong field environment of black holes and other compact objects, and the state of matter at supra-nuclear energies in neutron stars"

Black holes The 100 micro arc second resolution of Luciola at $500 \mathrm{~nm}$, and 20 micro arc second at Lyman alpha, is of interest for imaging the close 
environment of black holes such as GRS $1915+105$, the binary structure which they often have, the accretion disks and jets.

Microquasars These galactic binary X-ray sources, exhibiting jets with relativistic or super-luminal velocities, are observable from the gamma to the radio range $[6,28]$. They are believed to contain a black hole and a stellar companion feeding an accretion disk. The angular resolution of Luciola, down to 20 micro arc seconds in the $200 \mathrm{~nm}$ ultra-violet, is of considerable interest on SS 433, Sco X1 and other microquasars to clarify the connexion of the jet and accretion disk.

Neutron stars On neutron stars, much longer baselines, approaching 100,000 kilometers, would be needed to resolve the $20 \mathrm{~km}$ size expected for the star itself. This should later become feasible with a very large "Neutron Star Imager" hypertelescope, but Luciola can already image the environment of neutron stars. The binary pulsar J0737 3039 studied by Hulse and Taylor has $700,000 \mathrm{~km}$ spacing at an estimated distance of 600 parsecs. Its corresponding angular spacing 7.7 microarcsecond is nearly resolved by $1 \mathrm{~km}$ baselines at the $120 \mathrm{~nm}$ ultra-violet limit, and a rotating elongated image may be detectable if both components emit significantly at this wavelength.

\subsection{The early universe}

Lensing, micro-lensing and diffractive lensing Arc and duplicity patterns of gravitational lensing and micro-lensing carry rich information on the lensing body and the background source, which is already exploited for probing dark matter and dark energy. The high resolution of Luciola can contribute to this effort.

The possible dark matter component which may be present in the form of dark bodies, such as lost planets or single black holes in the interstellar space, has yet eluded detection efforts. But such bodies, if they exist at sub-parsec distances from the Sun, may become detectable from the occasional brief pulses of diff ractive micro-lensing occurring when aligned with background stars in external galaxies [5]. No such pulses have yet been observed, but they may become observable with Luciola. Unlike conventional micro-lensing events, which have never been recurrent on a given lensing mass, diffractive micro-lensing events are expected to repeat at months intervals on a diffractive micro-lens which is detected. The related lensing effect described by Gould and Gaudi [11] is also of interest to reach extremely high resolution.

The early optical emission of gamma-ray bursts, during the first minutes after receiving alerts, may occasionally be observable when occurring within the broad primary field of the upgraded Luciola, if it has a focal spaceship equipped with strong thrusters. 


\subsection{The universe taking shape}

"Find the very first gravitationally bound structures that were assembled in the Universe - precursors to today's galaxies, groups and clusters of galaxies - and trace their evolution to the current epoch"

Luciola's capability to image complicated deep fields containing many very faint "active resels" (Fig. 3), for example in the form of faint galaxies, is applicable to cosmological deep fields. The spectroscopic information simultaneously obtained gives redshifts of remote galaxies.

\subsection{The evolving violent universe}

"Trace the formation and evolution of the super-massive black holes at galaxy centres - in relation to galaxy and star formation - and trace the life cycles of matter in the Universe along its history"

Active galactic nuclei, in the form of Seyfert galaxies, quasars, and other violent sources containing accretion disks have very small angular sizes relevant for high-resolution imaging with Luciola. They are believed to contain a supermassive black hole, or several of them as a consequence of galaxy mergers [19]. In such cases, much useful information can be gained with high-resolution imaging, as demonstrated for our galaxy by recent observations which gave measurements of the central invisible mass from the fast motion of orbiting stars.

\subsection{Other science}

In addition to the Cosmic Vision targets, Luciola can provide much insight into the physics of stars, including many types of poorly understood atypical stars, single, multiple or clustered, by imaging their surface and environment such as accretion disks, jets, planetary nebulae, etc... Snapshot spectro-images of star surfaces can greatly improve our understanding of their physics, especially with regard to magnetic activity and dynamo theory. Multiple stars, with their mass loss, accretion disks, and occasional jets are natural laboratories which can be resolved in many cases by Luciola. In the near and mid infra-red ranges, the dust structures are also imageable. Globular clusters are also particularly interesting targets.

\subsection{Possible convergence with the NASA Stellar imager}

Also, the science program of the NASA Stellar Imager (SI) project [18] can in principle be tackled by Luciola if upgraded with an SI focal spacecraft, assumed built to Luciola standards by NASA with the SI team. The SI science and possible synergy are described by the SI team leader, Dr. Kenneth Carpenter, as follows: "We note that there are many similarities in science goals and observatory architectures between Luciola and the NASA Stellar Imager 
(SI) "Vision Mission" concept (http://hires.gsfc.nasa.gov/si/). SI is envisioned as a UV/Optical, space-based Fizeau interferometer to enable 0.1 milli-arcsec (mas) spectral imaging of stellar surfaces and, via asteroseismology, stellar interiors and of the Universe in general. It has a reconfigurable sparse array of 30 1-m primary mirror elements, with a maximum baseline adjustable from 100 to 1,000 m, which focus source light on a beam combiner located from 1 to $10 \mathrm{~km}$ distant. Although the current design of SI uses a different architecture, a credible alternative is in fact a "hypertelescope" design like Luciola. In either design case, SI requires the development of precision formation flying of $\sim 32$ separate spacecraft and closed-loop optical control of $\sim 30$ mirrors, both technologies which are also needed for Luciola. There are thus possibilities of enormous future synergies between the two projects.

Beyond these synergies, there is also the long-term possibility that the two concepts might converge into a single facility that would do the science of both programs at significantly lower cost and total effort than would be required for two independent projects. If this were to happen, then one could envision SI/NASA contributing a specialized beam-combiner (focal) spacecraft to a ESA-built Luciola, with its own specialized beam-combiner spacecraft. As pointed out in various SI studies (e.g., [27], "The Stellar Imager(SI) Vision Mission”, in SPIE Astronomical Telescopes and Instrumentation, May 24-31, Orlando, FL, SPIE Paper \#6268-77), having two different "hub" (beamcombiner) spacecraft adds greatly to the efficiency and reliability of the observatory by enabling a pre-positioning of the second hub while the first is being used for observation and by providing redundancy of a critical-path element without which the observatory would fail. Another possibility is that ESA and NASA might both build components of the primary array to enable an array with more components than might otherwise be possible.

Although, like Luciola, SI has not yet progressed beyond the concept development stage (i.e., it has not yet been "approved" by NASA for formal development or flight), it is included as a "Flagship and Landmark Discovery Mission" in the 2005 NASA Sun Solar System Connection (SSSC, now the Heliospheric Sciences Division) Roadmap and as a candidate for a "Pathways to Life Observatory" in the NASA Exploration of the Universe Division (EUD) Roadmap (May, 2005), and as such, does continue to be developed as a candidate for far-future flight approval. The inclusion of Luciola in the ESA Cosmic Vision plans would thus facilitate future possible and important collaborations with the SI Development Team that could prepare both concepts for further convergence as discussed above."

\subsection{High resolution astrometry}

Another science area which appears practicable with a suitably upgraded Luciola is astrometry with extreme angular accuracy, using two or more specialized focal spacecraft located several degrees apart in the broad primary field. For a very accurate calibration of the angle measurements thus achievable, the spacing of these spacecraft in the primary field is measured 
by laser interference, with a laser connexion between them. In addition to the astrometric search for exoplanets, such Luciola astrometry is applicable to the detection of variable distorsions in the pattern of remote galaxies, caused by the gravitational lensing effect of foreground masses, including undetected black holes, lost planets, etc...

Additional science targets of interferometry, however not including the prospect of rich snapshot imaging with hypertelescopes, are also listed in the proceedings of the recent NOAO Workshop "Future Directions for Interferometry" (2007).

\subsection{Scaling laws for science and cost}

The science yield when observing faint and complex objects varies approximately as the number of active resels (for example stars in a cluster) simultaneously imageable, which itself varies as $N^{2}$, and as the base-2 logarithm of the signal/(photon noise) ratio (this $\log _{2}$ dependance, suggested by the referee in accordance with information theory, is adopted here instead of the linear dependance used in the original proposal), amounting to $N^{5 / 4} P_{\mathrm{t}}^{1 / 2}$ [31]. Pt is the total number of photons detected during the observation, varying as the collecting area $N d^{2}$. The signal/(photon noise) ratio therefore varies as $N^{7 / 4} d$, and the product describing science as $\mathrm{Sc}=N^{2}\left\{7 / 4 \log _{2}(N)+\log _{2}(d)\right\}$. The cost $C_{\mathrm{pa}}$ of the collector flotilla can be coarsely evaluated as $C_{\mathrm{pa}}=N d^{\gamma}$, where the $\gamma$ exponent may have a value between 2 and 3, depending if the cost is proportional to the area or volume and mass of the collector flotilla. By eliminating $\mathrm{N}$ between both expressions, the amount of science is thus found to vary as:

$$
\mathrm{Sc}=C_{\mathrm{pa}}^{2} d^{-2 \gamma}\left\{(7 / 4) \log _{2} C_{\mathrm{pa}}+(1-7 \gamma / 4) \log _{2} d\right\}
$$

It can also be expressed as a function of $N$ :

$$
\mathrm{Sc}=N^{2}\left\{(7 / 4) \log _{2} C_{\mathrm{pa}}+(1 / \gamma-7 / 4)\left(\log _{2} C_{\mathrm{pa}}-\log _{2} N\right)\right\}
$$

As an example, if $\gamma=2$ the science is increased 20,000 times if a collecting area of $30 \mathrm{~m}^{2}$ is made of 3,000 mirrors of $10 \mathrm{~cm}$ rather than 30 mirrors of $1 \mathrm{~m}$. If $\gamma=2.5$, the science gain is 200,000 with the $10 \mathrm{~cm}$ mirrors, now numbering 95,000 and providing $95 \mathrm{~m}^{2}$ of collecting area while the number, size, and total collecting area of the $1 \mathrm{~m}$ mirrors have remained invariant.

At given collector cost, the amount of science therefore increases very fast if the sub-aperture diameter is reduced. Such reduction, affecting the mass of a nano-satellite as $d^{3}$ and its solar cross-section as $d^{2}$, also improves as $1 / d$ the acceleration of the optional solar or laser propulsion applied to the nanosatellites, and therefore decreases as $d^{1 / 2}$ the time spent in repointing, which further increases the science. 


\section{The Luciola hypertelescope concept}

Luciola has a kilometer-sized diluted mirror, materialized as a formation flight of many nano-satellites carrying small mirror elements, with a focal combiner spacecraft exploiting narrow pieces of the broad sky image focused by the diluted mirror along its focal surface. There is also a metrology and communications spacecraft located at the curvature center of the mirror flotilla. The instrument is expected to operate initially with 12 collector elements at least, and a single focal spacecraft. Successive upgrades, at intervals of a few years, increase the aperture count to 100 and then 1,000, while the number of focal spacecrafts will increase to 3 or more, and then perhaps many more if other space agencies build such spacecraft to better exploit the broad sky image focused by the collector array. Multiple focal spacecraft thus observing different targets in parallel multiply the science yield from the basic investment made in the form of the collector array, metrology spacecraft, etc... Some of them can be dedicated to specific observing modes and have different focal instruments.

As discussed in Section 2.11 and recent articles [10, 30-34], the reason for using many small apertures rather than a few large ones is that it greatly increases the science yield by increasing the information content in snapshot images. In terms of engineering, it reduces the risk in several respects and relaxes the reliability requirements while favouring the interchangeability of elements and allowing a maintenance programme for a long service life. With many spacecraft, a few disabled ones do not greatly affect the array performance, turning the system an extremely robust one. Indeed with constellation replenishment involving only very light and robust nano-satellites, Luciola can be considered a permanent facility whose focal plane instruments will independently evolve and be increased in number over time, The observatory philosophy considered, and the continuing expansion of the optics through periodic up-grades, suggest that it can be exploited and improved for several decades. It is indeed designed as a modular system where aging and obsolescence can be avoided by delivering replacement spacecraft during upgrade missions, also programmed to greatly expand the observing efficiency. Such upgrades are expected to allow a continuing growth of the observatory.

For matching the requirements of the varied science targets mentioned above in Section 2, the Luciola hypertelescope is also designed to be easily reconfigurable, in terms of aperture pattern and resolution, in a matter of hours or days according to the size and morphology of the sources to be observed.

\subsection{L2 halo orbit}

Halo orbits around the L2 Lagrange point of Sun-Earth are particularly suitable. Their exceptionally uniform micro-gravity, and ensuing low tidal forces, is indeed compatible with the weak accelerations of solar propulsion, if the option is adopted for the collector spacecraft. To prevent the collector flotilla from continually drifting away from the sun, in response to its radiation 
pressure exerted upon the low mass nano-satellites, its location can be slightly offset toward the sun for a neutral photo-gravitational buoyancy. The more massive satellites serving in the focal plane and for metrology can also be equipped with proportionately large solar sails or photovoltaic panels, about $5 \mathrm{~m}$ in size if the mass is $100 \mathrm{~kg}$. to achieve a matching buoyancy. Alternately, micro-thrusters applying milli-Newton forces can similarly maintain the balance, at the expanse of fuel. As remarked by the referee, the weak thrust of the presently available thruster systems may affect the reconfiguration time. A tradeoff should then be found between: a - the ratio of "observing" versus "reconfiguration" times; b - the total duration of the mission; c - the given initial reserve of propellant (expressed for example in $\mathrm{m} / \mathrm{s}$ ); $\mathrm{d}$ - the total number and repartition of the astrophysical targets observed; and e - the wet mass of the combiner spacecraft.

Following successful operation with the initial configuration for a few years, upgrade missions are planned for delivering additional spacecraft to the collector array and/or the focal surface. If it becomes justified, a complete diluted sphere of nearly static collector mirrors can be progressively built-up for panoramic observing.

\subsection{Impacting micro-meteorites: recovery of position and attitude}

The low-mass nano-satellites of the collector array are easily disturbed by micro-meteorite impacts. They may survive impacts with the most abundant micron-sized grains, but their attitude can be disturbed, with a spinning motion. The passive self-repointing capability of the solar propulsion optics is then expected to regain the control of attitude and position within hours or days. Estimates based upon Grün's distribution of the micro-meteorite flux vs. size indicate that disturbing impacts on the typical $0.1 \mathrm{~m}^{2}$ cross section of a Luciola nano-satellite will occur sufficiently rarely to keep most collector elements operating at any time.

\subsection{Optical design}

The formation-flying nano-satellites of the collector flotilla each carry one element of the diluted collector mirror (Fig. 5). These elements are nearly flat concave mirrors, with focal length $\mathrm{F}=3,600 \mathrm{~m}$. The flotilla accurately maintains its optical geometry, under control from the metrology spacecraft located at the curvature center, and is globally pointed and repointed to science targets, although the independant motion of the focal spacecraft within the broad primary field of the collector array gives them much freedom to observe local objects.

The collector diluted mirror can be paraboloidal or spherical, each option having its advantages and drawbacks, some of which were previously discussed for ground-based ELTs. The proposed upgrade plan involves a paraboloid as the initial configuration of the diluted collector, then slightly $(10 \mathrm{~mm})$ reshaped to a sphere when additional beam-combiner spacecraft are delivered. This 


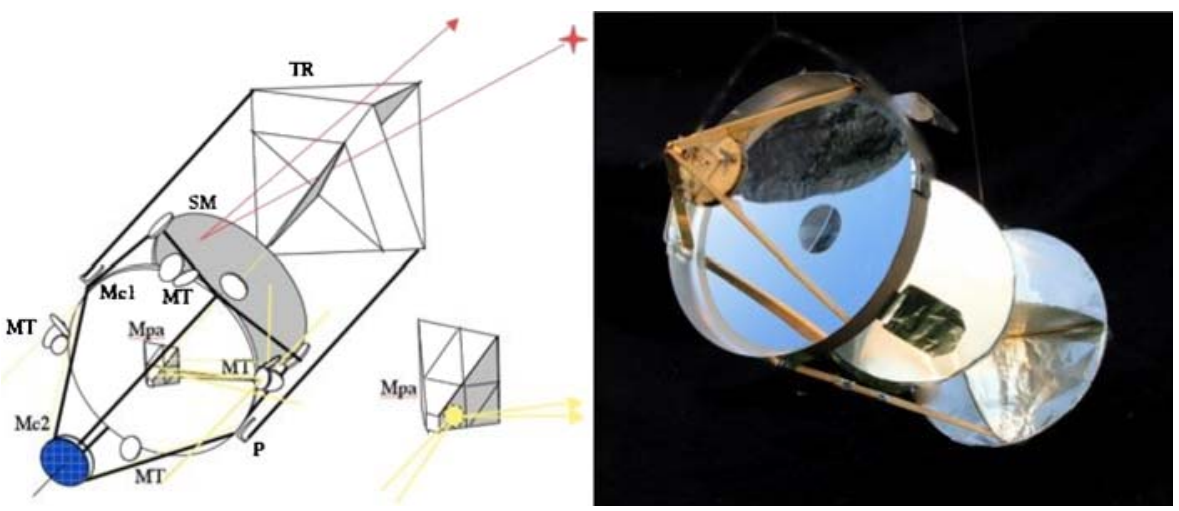

Fig. 5 Unitnano-satellite of collector flotilla with the optional solar propulsion system. This system includes a solar concentrator in the form of a Gregorian telescope with collector mirror Mc1 and secondary mirror Mc2. The latter is controlled in tip and tilt by a pair of actuators, so that the sun's image, once coarsely pointed, can be directed toward any of 16 reflective facets arranged as a pyramid (inset at right). Each facet is concave and relays a pupil image on a corresponding small mirror MT at the edge of Mc1. Twelve such mirrors are variously oriented to reflect the solar light in different directions, and four additional radial directions are directly fed from corresponding facets of the central pyramid. Modulating the tip-tilt actuators of Mc2 can thus activate all six force and torque components needed to drive the position and attitude of the nano-satellite. The stellar mirror SM is located in the shadow of the solar propulsion mirror Mc1, and rotated about one axis by a micro-motor, the second pointing axis being controlled by global rotations of the nano-satellite about the sun's direction. A retro-reflective tail TR, becoming illuminated by sun light when the solar pointing of Mc is lost, serves to restore this pointing. Angular dampers, not shown, absorb the oscillation energy. The entire structure has minimal mass, a few hundred grams for a $25 \mathrm{~cm}$ stellar mirror, for maximal accelerations in response to the solar propulsion

slight global reshaping does not require replacing the mirrors if their size is small enough to fit either global surface, within Rayleigh's tolerance. Such is the case at ultra-violet and visible wavelengths with a $1 \mathrm{~km}$ collector mirror at $\mathrm{F} / 3.6$, if the segments are $25 \mathrm{~cm}$ in size or smaller. But a corrector of spherical aberration must be attached to the initial focal satellite, when upgrading the global collector figure from paraboloidal to spherical.

Phasing the sub-apertures with piston actuators Positioning errors among the collector mirrors affect the phasing of the combined beams and therefore the formation of the interference peak. Using the redundant wave sensor signals from a star and from the central metrology laser, discussed in Section 3.3.3, tiptilt and piston corrections can be made either at the collector mirror segments or within the focal spacecraft, in a relayed pupil plane. The former method is preferable for the upgraded Luciola having several focal spacecraft, since each then does not have to be separately corrected if its internal optics is perfect. Unless the solar propulsion system, a possible option for driving the collector nano-satellites, itself proves accurate enough, at the $100 \mathrm{~nm}$ level, to serve as a micro-motion stage, three additional actuators are needed for carrying each collector mirror. This can be decided after a prototype nano-satellite with 
the solar propulsion system will be tested in a "bird cage" outside the ISS, where its positioning response can be accurately measured with laser fringes. If additional fine actuators prove necessary, they may be inserted on the back side of each collector mirror, in the form of piezo elements or voice coils for example.

Sub-nanometer piston corrections are needed for "extreme" visible coronagraphy, unless applied at the Lyot stop [25] where their accuracy can be much relaxed. Such ultra-fine corrections should be made within the "Visible Planet Imager" focal spacecraft equipped for coronagraphy.

Retro-reflectors Each collector mirror carries on its rim three small retroreflectors, equi-spaced and having their vertex accurately positioned to match the mirror surface. These can be hollow-type corner cubes or cat's eye designs based on small lenses or mirrors. Their acceptance cone should be very broad, and if possible fully panoramic, to accommodate the strong attitude errors occurring occasionally after meteorite impacts or other malfunction. Filters can be attached to them for spectral tagging, of interest if the metrology optics at the laser source does not angularly separate the retro-reflectors within a triplet. Additional reflectors can be attached to the solar thruster head to monitor its orientation.

\subsubsection{Focal beam-combiner spacecraft}

Figure 1 shows the beam-combiner optics, without the corrector of spherical aberration needed upstream if the collector is spherical. It has relay optics with micro-optical arrays providing many $(10 \times 10$ for example) parallel imaging channels, each producing a high-resolution direct image of a narrow field of view within adjacent "sky lobes" of diffraction through the sub-apertures. With collector mirrors of $25 \mathrm{~cm}$ size, the lobe extent is 0.5 arc second at visible wavelengths, corresponding to $9 \mathrm{~mm}$ in the focal plane at $\mathrm{F} / 3.6$. In comparison with conventional interferometers having few sub-apertures, considerable science information is gained in the rich direct images, and with the numerous fields simultaneously imaged. One or more channels are equipped with coronagraphic optics for imaging exoplanets.

The images in each channel are spectroscopically analyzed, with spectra recorded for each of their resels, using a faceted field grating in the relayed image plane. This spectro-imaging information, recorded in the form of parallel spectra by the photon-counting science camera, is also exploited to extract piston error signals, using the "dispersed speckle" algorithm [26]. This is redundant with the laser metrology measurements of piston errors, also performed continuously during the observation, but provides a useful verification and accurate calibration of minor differences or drifts which may arise in the non-common light path, i.e. the focal optics.

Within the initial beam-combiner spacecraft, at the focus of the paraboloidal collector, the size of the diffraction-limited field is limited by paraboloid coma to a very small angle, 64 milli arc second in the visible at F/3.6. For exploiting 
several adjacent imaging channels, located 0.5 arc second apart in the visible, those located off-axis need individual coma correctors in their tiny relayed pupils, at the entrance pupil of each densifier.

The coma aberration of the paraboloid also precludes using additional focal spacecraft off-axis, unless the primary focal ratio is much reduced, in which case deformable correctors can be used, according to the F/10 hypertelescope design proposed by Dohlen et al. [21].

\subsubsection{Aperture reconfigurations}

The direct image within a DIF channel can in principle be optimized in different ways:

A By varying the pupil densification factor, which keeps the angular resolution invariant but inversely varies the DIF extent and the image luminosity. This is achievable if the micro-optical pupil densification elements are zoomable. On a compact science target such as a resolved star or small galaxy, the pupil densification should be increased until the object at least fills the DIF, for maximal intensification. Further shrinking the DIF causes windowing in the object's direct image. But a full "stitched" image of the object can then be reconstructed from successive exposures made with slightly offset pointing. Both methods have equivalent photon efficiency, but the second can be preferable in the infra-red with a non photon-limited detector.

B By varying the global size of the collector array, which also zooms the image and varies its angular resolution.

C By changing the aperture pattern, according to the object morphology. Non-redundant configurations are of interest for improving the resel count in direct images, as well as the crowding limit on complex extended objects. Apodized aperture patterns (Figs. 6 and 3 ) are of interest for high dynamic range imaging. Periodic configurations can be of interest for coronagraphy since they allow a full densification of the exit pupil, then exploited like that of an ELT mosaic.

The focal optics sketched in Fig. 1 can accommodate a series of predefined aperture patterns, periodic or non-redundant, if the micro-scale pupil densifiers in the AP array are interchangeable. A conventional wheel-type mechanism carrying a set of such components can thus accommodate reconfigurations of the collector flotilla geometry and size. Continuous zooming, while the collector flotilla is expanding or contracting, is also achievable if zoom features are incorporated into the $\mathrm{AD}$ and $\mathrm{AP}$ arrays, which is feasible in principle if the plates are double, with adjustable spacing and axial position.

The focal spacecraft must also be capable of stronger accelerations than the collector nano-satellites when the hypertelescope flotilla is globally re-pointed by rotating about the vertex of the collector paraboloid. This is especially needed when receiving alerts of fast transient phenomena such as gamma ray bursts, supernovae, etc.... Given its higher mass, hundreds of kilograms, 

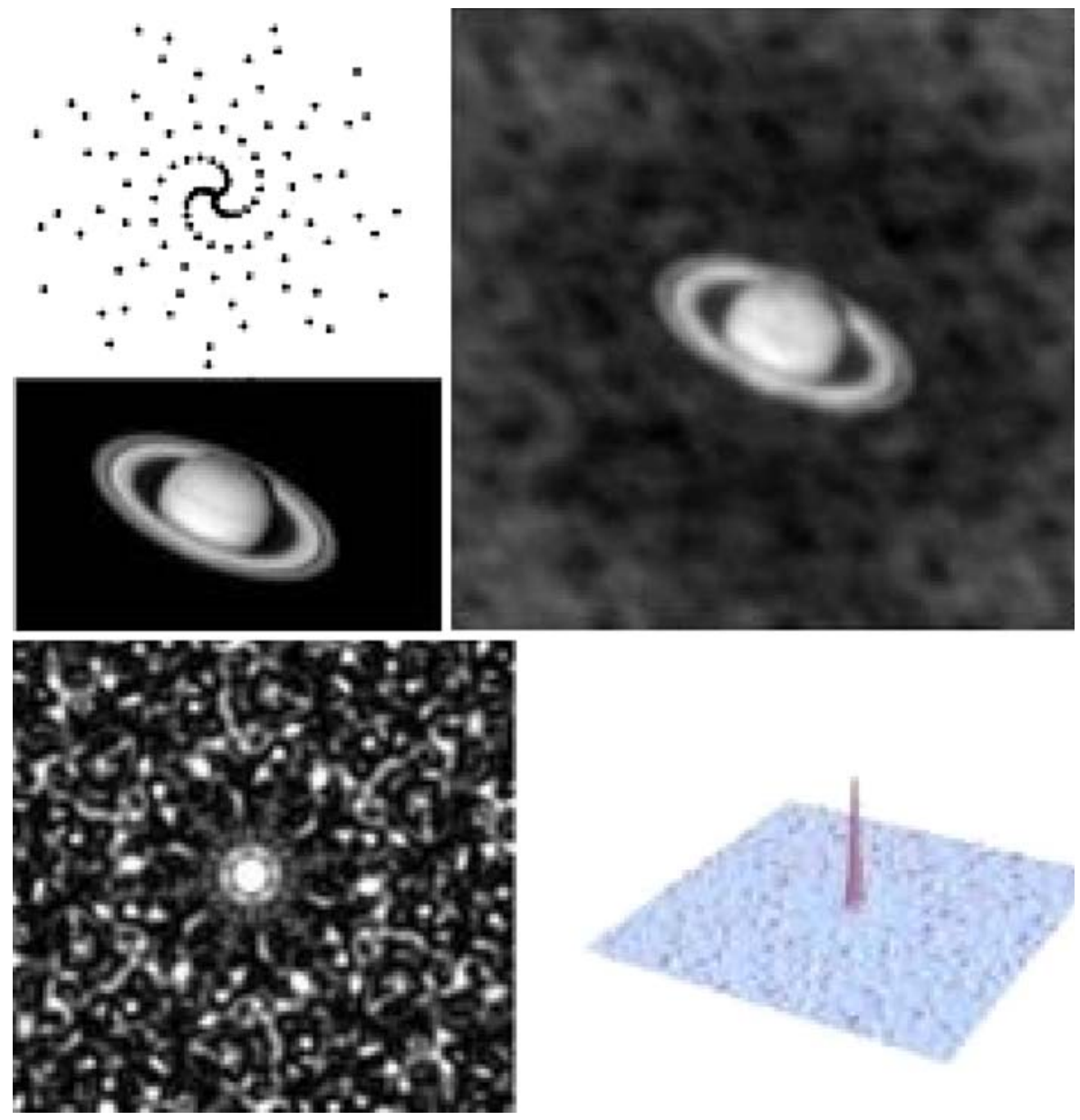

Fig. 6 Simulated direct image (top right) with 106 apertures (aperture pattern at top left), obtained by convolving a Saturn picture (middle left) with the spread function (bottom left and right). No aperture rotation was used and the image is unprocessed

solar propulsion would require large sails, and they could cause unwanted obscuration in the collected starlight. Applicable types of stronger thrusters are FEEPs and cold gas, already validated by ESA.

Size and mass The focal beam-combining spacecraft is sufficiently large to carry a metre-size instrument with tens of kilograms of payload, mostly in the form of micro-optical and camera components, and also the corrector mirrors in the upgraded versions. Keeping their size moderate is what limits the collector's focal ratio to about F/3.6.

Standard spacecraft platforms qualified for operation at L2 are probably adequate, once equipped with the appropriate optical payload. The position 
and attitude tolerances are relaxed, with respect to the collector elements, and amount respectively to $0.1 \mathrm{~mm}$ and 10 arcseconds. The axial position however remains critical since it affects image focusing. The tolerance is $2 \mu \mathrm{m}$ at F/3.6 in the visible, but fine focus control can be incorporated in the adaptive mechanism within the optical package.

\subsubsection{Metrology and communication spacecraft at curvature center}

A spacecraft at the curvature center of the collector flotilla can control its optical geometry with extreme accuracy, using laser interference techniques which have been developed and demonstrated for the Carlina-1 ground-based precursor. Techniques developed for the NASA Stellar Imager at Goddard Space Flight Center are also relevant.

The metrology optics (Fig. 7) has one or more alignment cameras and metrology devices involving one or more diode lasers, operating at several wavelengths or in white light for absolute distance comparisons. The spacecraft also carries a computer, and communication electronics which emits signals toward each collector element to direct its positioning and attitude. It also sim-

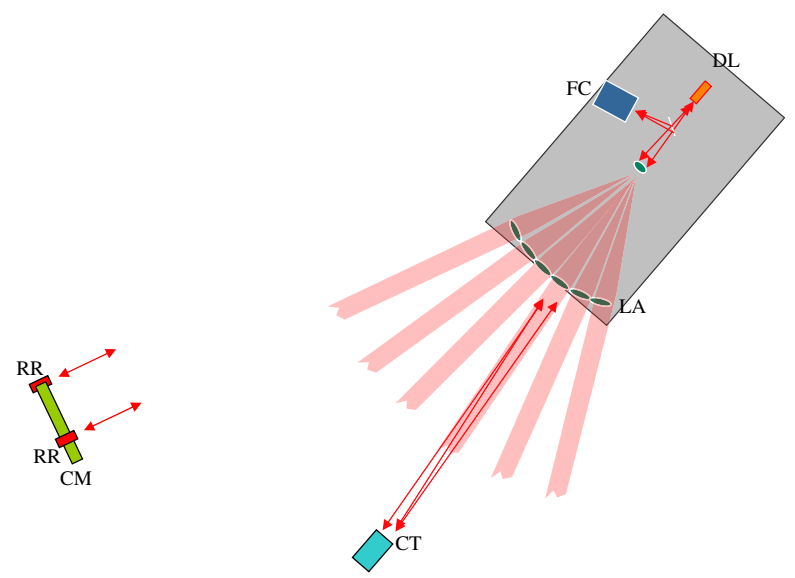

Fig. 7 Opticsof the metrology spacecraft at the curvature center. A pulsing polychromatic diode laser DL, collimated with a lens array LA, launches a fan beam toward the flotilla of collector mirrors $\mathrm{CM}$. Light returning from their retro-reflectors $\mathrm{RR}$ reaches the fast camera FC, and timeof-flight measurements give the distances with millimetre accuracy. A refined accuracy, down to nanometers, is obtained with interference measurements using, as a reference wave, light reflected from the focal station, with double-pass (interferometer detail not shown). The camera $\mathrm{C}$ resolves the triplet of retro-reflectors attached to each collector mirror, so that tip-tilt errors be accurately measured with the interference fringes. Several interchangeable lens arrays LA are provided for accommodating reconfigurations of the collector flotilla. With the paraboloidal collector figure used initially, there is a $2 \times 10 \mathrm{~mm}$ optical path difference among the beams, which is accomodated by spectrally modulating the laser, or by adding a matching aspheric corrector on the reference wave. A star-tracker camera CT sees the collector flotilla together with the background stars, thus providing error signals for the transverse positioning of each nano-satellite, with the required centimetric accuracy 
ilarly controls the focal combiner spacecraft to retrieve the camera images and other diagnostic data such as the redundant piston measurements extracted from the science images. It requires one- or two-way communication links toward the collector nano-satellites and the focal spacecraft, in addition to the Earth data link which is also preferably located at the curvature center.

\section{Propulsion of collector nano-satellites}

Current studies of formation flying by NASA and ESA have considered different propulsion options. Among these are cold and hot gas jets, ion engines, colloidal jets. Cold gas is demonstrated with the four SPHERES elements aboard the ISS. These options are also to be considered for the Luciola flotilla, where the axial position of each flyer must be controlled with sub-micron, or only millimeter, accuracy depending if an additional stage of fine piston actuators is to be used. The accelerations needed for repointing the instrument are moderate, especially if there is a wide collector field where one or several more agile focal combiners can speed up the star acquisition.

\subsection{Option of solar propulsion for the collector nano-satellites}

Since 1982, some of us have investigated solar propulsion schemes suitable for elements of a formation-flying interferometer. Figure 5 shows our current concept of solar propulsion for the low-mass collector nano-satellites, developed with numerical simulations and tested in the laboratory with models suspended from a torsion wire, under solar illumination. In spite of the weak accelerations which it can provide with even low-mass flyers, it is expected to have advantages of cost, reliability, long life, and no contamination of the cold optical surfaces by exhaust plumes. Following additional laboratory testing, the concept however requires validation in space, aboard the ISS.

The very weak radiation force exerted on a mirror by sunlight, amounting to $2 \mathrm{P} / \mathrm{c}$ at normal incidence if $\mathrm{P}$ is the power received and $\mathrm{c}$ the speed of light, can be harnessed to apply all six components of force and torque needed to translate a nano-satellite and control its attitude. The solar acceleration increases if a structure is scaled down since its mass decreases as the cube of the scaling factor, while its cross-sectional area decreases as its square.

As shown in Fig. 5, each collector mirror element is carried by a nanosatellite driven by a compact solar sail. It is configured as a small Gregorian telescope, normally pointed to the Sun, with output switchable in 16 different directions by tilting the Gregorian secondary mirror. Modulating the exit direction can produce any average combination of torques and forces. The primary Gregorian mirror also serves as a sunshield which passively cools the stellar collector mirror located in its shadow, a scheme similar to the JWST.

The solar radiation pressure is slightly less than a microNewton on a $25 \mathrm{~cm}$ mirror, but the response is expected to be highly linear and predictable, with no stick-slip, hysteresis, travel limitation or other problems of earthly actuators. 
In the absence of wind and the other disturbances also affecting Earth-based interferometers, it is therefore expected that the solar propulsion system can maintain the position of the stellar mirror within microns, and possibly better if the vibrations of the small Gregorian solar mirror can be decoupled from the stellar collector mirror. If it can be controlled within $100 \mathrm{~nm}$, then additional piston actuators are unnecessary.

The tip-tilt actuators of mirror Mc2 can be electrostatic, or small solenoids, or piezo elements. Feedback may involve light detectors between the facets of Mpa. Power is provided by a small solar cell attached to Mc2, or larger ones around $\mathrm{Mc1}$, and batteries are probably not needed since the spacecraft is passively self-repointing towards the Sun. For maximal response in terms of solar acceleration, the whole structure is expected to have a very low mass, down to a few hundred grams for a $25 \mathrm{~cm}$ stellar mirror. The on-board electronics is expected to be very simple, and should therefore not contribute much to the mass budget. The solar mirrors Mc1 and Mc2 have a modest optical quality and can be made of stamped aluminum sheet, $100 \mu \mathrm{m}$ thick and electrolytically polished.

Instead, the optical quality of stellar mirror Ms1 must meet the Rayleigh tolerance. Thin SiC honeycomb is a candidate material, as well as silica aerogel, molded or diamond turned.

At right: Laboratory testing model of a collector nano-satellite with solar propulsion actuator in the form of a $25 \mathrm{~cm}$ Gregorian telescope (visible at left), $17 \mathrm{~cm}$ stellar mirror (center) and retro-reflective tail (right). Only two of the twelve small peripheral solar mirrors are installed. The model is suspended from a thin torsion wire to test the attitude control and self-pointing under solar illumination. The solar collector and secondary mirrors are made of thin electroformed nickel.

Self-repointing to the Sun and angular damping The solar propulsion system of the collector nano-satellites is expected to be self-pointing towards the sun, by passive means. During the initial deployment, and following occasional impacts by micrometeorites, this is necessary to bring the sun's image within the usable field of its Gregorian solar concentrator. It is achieved by the radiation pressure applied upon the retro-reflective tail, which becomes exposed to sunlight in the event of a large pointing error.

Oscillations, the period of which can amount to tens of minutes, are damped out with inertial masses, coupled with inelastic flexure joints. One option is to use the tail itself as a damped pendulum, attached with such joints. Some of us developed a numerical model of self-repointing using this option, with optical ray trace providing accurate values of the forces and torques at various attitudes.

The numerical simulation results confirm the damped self-repointing of the nano-satellite to the Sun. They have also shown that the concavity of the primary solar mirror induces unwanted stable attitudes at oblique angles relative to the Sun, where the skew focused beam misses the secondary mirror. This relates to the fact that a dihedral mirror pointing the sun is stable if shaped 
like a sun-illuminated roof, and unstable in the opposite case. Adding smaller reflectors or a conical reflective rim at the edge of the concave mirror restores the stability.

\subsection{Option with laser-driven mirrors (addendum to the Luciola proposal)}

The theoretical gain with smaller sub-apertures justifies efforts toward deploying a large number of miniature mirrors. Of interest is the concept of lasertrapped mirrors $[35,36]$, which can, in principle, be implemented as sketched in Fig. 8. A semi-transparent mirror, if non-absorbing and symetrically illuminated by a pair of coherent laser beams, can direct the emerging light toward
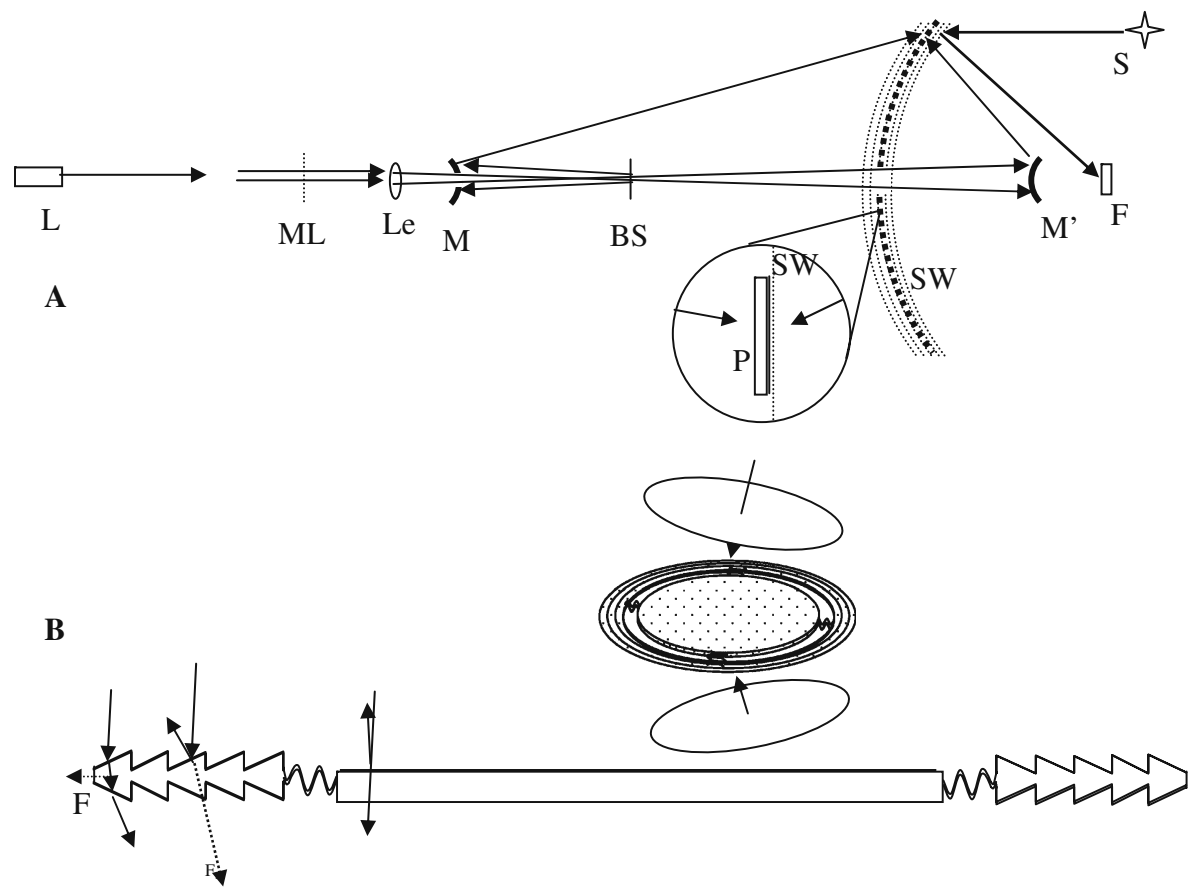

Fig. 8 Architecture for laser-driven collector mirrors. a Light from a white laser $\mathrm{L}$ is focused by lens Le and divided by beam-splitter BS to illuminate convex mirrors $\mathrm{M}$ and $\mathrm{M}$ '. They reflect counter-propagating beams which interfere in the form of a standing wave SW (thin dotted lines, and enlarged detail below). By suitably shaping $\mathbf{M}$ and $\mathbf{M}$ ', its shape can be made paraboloidal or spherical. Its radiation pressure tends to trap, axially, the hypertelescope's collector mirrors $\mathrm{P}$ (fat dotted line and enlarged detail below) if made in the form of small semi-transparent mirrors with matching shape. They focus light from stars such as $\mathrm{S}$ toward the beam-combiner spaceship F. b For transverse trapping, the laser light is concentrated on each mirror P by a micro-lens array ML, and the mirrors have a light-deflecting edge ring. Prismatic grooves deviate the light and apply its radiation pressure $\mathrm{F}$ outward, thus tending to center the mirror within the laser beams. For a coarse stabilization of the mirror's attitude, the grooves have a dichroïc coating, which can be made reflective or transparent by changing the laser's wavelength. When reflective (ray trace at right), the radiation pressure $F_{\mathrm{a}}$ is sensitive to the angle of incidence and the resulting torque tends to stabilize the mirror's attitude 
one or the other side, depending on its axial position, which defines the phase of each reflected wave. Its interference with the transmitted wave, on each side, is thus position-sensitive in terms of the intensity distribution among both emerging beams. A trapping situation is thus generated by the reversing radiation pressure. The periodic trap becomes confined to a single standing wave sheet if the laser emits white light. The trapping accuracy in the axial direction can be sub-wavelength, since the period of standing waves is $\lambda / 2$ in a pair of counter-propagating beams.

The exact figure of the giant diluted mirror being accurately defined by the standing wave, the laser system is, in a sense, doing the metrology, the actuation and the serving, but a damping mechanism is required to avoid oscillations. Also, it is of interest to trap the mirrors transversally, with lesser accuracy, and this is achievable with the "laser tweezer" effect if the illumination is concentrated on them, as shown in Fig. 8. Oscillations, axial and transverse, are damped passively by an inelastic flexure joint attaching the mirror and ring. Active damping also appears achievable, using wavefront sensing in a star's image and phase actuators for each laser sub-beam.

Laser-driven collector mirrors being small and passive, their unit cost can be low especially if mass-produced with molding or micro-electronic techniques, and their number very large. Extra spaceships are needed, in addition to the swarm of small mirrors, to carry M, M' and BS. The laser L and ML can be integrated with BS in a single spaceship, but, if the main flotilla is in the Earth shadow at the L2 Lagrange point, L is preferably located far away (several thousand kilometers), in a separate sun-illuminated spaceship powered by solar cells.

As an example, a $1 \mathrm{~km}$ "laser driven hypertelescope" can exploit 10,000 miniature mirrors having a $30 \mathrm{~mm}$ diameter and $0.1 \mathrm{~mm}$ thickness. Its collecting area exceeds somewhat that of the Hubble Space Telescope, and it should exceed its limiting stellar magnitude, of the order of $\mathrm{mv}=30$. Per imaging channel, within each lobe of sky extent $\lambda / \mathrm{d}=4$ arc-seconds at visible wavelengths, a direct image containing $10,000 \times 10,000$ resels is obained if the aperture is non-redundant or rotated azimuthally during the exposure [37].

The radiation pressure from a $1 \mathrm{~mW}$ pair of laser beams illuminating one mirror, and fully emerging on one side, is $\mathrm{P} / \mathrm{c}=6.6$ picoNewton, accelerating the $0.2 \mathrm{~g}$ mass at $0.03 \mu \mathrm{m} \mathrm{s}^{-2}$. The axial oscillation period of the mirrors in their trap is $27 \mathrm{~s}$, and their axial escape velocity is $30 \mathrm{~nm} / \mathrm{s}$. In case of escape, for example under the impact of a nano-meteorite, a mirror can be recaptured by aiming laser beams from both sides. It takes $22 \mathrm{~h}$ to push the mirror across $100 \mathrm{~m}$, starting at rest, and 32 hours if half the time is spent decelerating, so as to reach zero velocity, by reversing the laser beam. The sky can be scanned along a great circle in $\mathrm{Tgc}=9$ days, allowing 20 such scans to cover the celestial sphere every 6 months.

For the infra-red, and even perhaps in the far-infra-red and sub-millimeter range, the technique of laser cooling [38] may prove applicable to keep the collector mirrors at temperatures below a few Kelvins, using active damping with piston actuators in the laser beams. If so, a multi-aperture version of 
the proposed SPECS interferometer [39] may conceivably use, instead of two large telescopes, many small mirrors trapped by a laser, in "laser-driven hypertelescope" fashion. The trapping laser would at the same time actively cool the mirrors to the specified 4 Kelvin temperature, using active phase control for damping the mirror's Brownian motion. A 1-km "hyper-SPECS" array would require sub-apertures of $10 \mathrm{~cm}$ at least to keep their diffraction lobe smaller than $6 \mathrm{~m}$ at the focal plane, if its focal ratio is $\mathrm{F} / 1$ and the longest wavelength $0.64 \mathrm{~mm}$. However, smaller mirrors and focal optics can be used if the pupil densification is first achieved among groups of mirrors at an array of intermediate foci, before the final densification at the global focus.

The limiting magnitude for detecting point sources against the sky emission would be the same as with a pair of large telescopes providing equal collecting area, but, if many-pixel detectors are available the direct imaging would greatly increase the science yield, as calculated in Section 2.11.

\section{Conclusions and future work}

The science yield of hypertelescopes, with their direct-imaging capability on faint and complex sources, increases sharply with more apertures of smaller size, at given collecting area. It greatly exceeds that of conventional interferometers relying upon aperture synthesis to reconstruct images by incoherently combining successive exposures.

The broad science which can be addressed with a first-generation hypertelescope in space raises challenging issues toward its construction. For the flotilla of collector mirrors, laboratory testing is under way with both the solar- and laser-driving options, pending further testing which can be performed outside of the International Space Station. The optical and dynamic aspects are also modelled numerically. This study work is also supported by the current work on ground-based versions having a similar optical design: a $30 \mathrm{~m}$ "Carlina" prototype is under construction, and a $200 \mathrm{~m}$ science version under study.

A possible convergence of Luciola is foreseen with the Stellar Imager (SI) concept of Carpenter et al. [27], studied by NASA: a focal spacecraft built according to SI specifications for its science program can be delivered at any time to the Luciola flotilla for joining its suite of focal spacecrafts, thus sharing the cost of the collector array. The convergent ideas and work, on both sides of the Atlantic, suggests that hypertelescopes will become a major evolutionary trend of future astronomy, with a vast growth potential toward $100 \mathrm{~km}$ and even 100,000 km apertures. The sub-millimeter radio range will also benefit from hypertelescope imaging as multi-pixel detectors become developed.

Acknowledgements We thank the unknown referee for his detailed suggestions toward improving the article, with respect to the version submitted as a proposal to ESA. Sebastien Morel suggested to explore laser driven mirrors. We also wish to thank for their encouragement all "supporting scientists": Jean Surdej, Philippe Stée, Swapan K. Saha, Francois Reynaud, Gerd Weigelt, Daniel Gezari, Christian Bracco, Sébastien Morel, Robert Woodruff, Dainis Dravins, Naoshi Baba, Guy Perrin, Mario Gai, William Danchi. 


\section{References}

1. Labeyrie, A., Lipson, S., Nisenson, P.: An Introduction to Optical Stellar Interferometry. Cambridge U. Press, Cambridge (2006)

2. Labeyrie, A., Schumacher, G., Savaria, E.: Flute or trio: different approaches to optical arrays in space. Adv. Space Res. 2(4), 11-22 (1982)

3. Labeyrie, A., et al.: 1984 TRIO: a kilometric array stabilized by solar sails. In: Proc. ESA Coll. Kilometric Optical Arrays in Space, Proc. ESA SP-226, pp. 27-33 (1985)

4. Stachnik, R.V., Gezari, D.Y.: 1984 SAMSI: an orbiting spatial interferometer for microarc second astronomical observations spacecraft array for Michelson spatial interferometry (SAMSI). In: ESA Coll. Kilometric Opt. Arrays in Space, Proc. ESA SP-226, pp. 35-42 (1985)

5. Labeyrie, A.: Gravitational lenses as giant diffractive telescopes. Astron. Astrophys. 284, 689 (1994)

6. Mirabel, F., Rodriguez, L.F.: A superluminal source in the galaxy. Nature 371, 46 (1994)

7. Léger, A., Puget, J., Mariotti, J., Rouan, D., Schneider, J.: How to evidence primitive life on an exoplanet? - The Darwin project. Space Sci. Rev. 74(1-2), 163-169 (1995)

8. Labeyrie, A.: Resolved imaging of extra-solar planets with future $10-100 \mathrm{~km}$ optical interferometric arrays. A\&AS Ser. 118, 517-524 (1996)

9. Damour, T., Esposito-Farnese, G.: Light deflection by gravitational waves from localized sources. Phys. Rev., D 58, 4 (1998)

10. Labeyrie, A.: ELTs, interferometers and hypertelescopes at different wavelengths. In: SPIE Conf., Lund (2007)

11. Gould, A., Gaudi, S.: Ap. J. 486, 687-692 (1997)

12. Labeyrie A.: Exo-earth discoverer, a free-flyer interferometer for snapshot imaging and coronagraphy. In: Proc. Extrasolar Planets: Formation, Detection and Modelling, Lisbon, 27 April1 May 1998 (1998)

13. Bracco, C., Teyssandier, P.: Scintillation in scalar tensor theories of gravity. A\&A 339, 921 (1998)

14. Labeyrie, A.: Snapshots of alien worlds - the future of interferometry. Science, Sep 17, pp. 1864-1865 (1999a)

15. Labeyrie, A.L.: Exo-earth imager for exoplanet snapshots with resolved detail. In: Proc. Conf. Working on the Fringe, Dana Point, USA, May 22-25 1999, PASP Conference Series (1999)

16. Boccaletti, A., Riaud, P., Moutou, C., Labeyrie, A.: Snapshot coronagraphy with an interferometer in space. Icarus 145, 628-636 (2000)

17. Labeyrie, A., et al.: Epicurus, a precursor hypertelescope. Proposal to ESA (2000)

18. Carpenter, K.G., Schrijver, C.J.: A dream of a mission: stellar imager and seismic probe. In: Proc. 196th AAS Meeting, June 2000, Bulletin of the American Astronomical Society, 32, p.721 (2000)

19. Milosavljevic, M., Merritt, D.: Formation of galactic nuclei. Ap. J. 563, 34-62 (2001)

20. Riaud, P., et al.: Coronagraphic search for exo-planets with a hypertelescope. I. In the thermal IR . Astron. Astrophys. 396, 345-352 (2002)

21. Dohlen, K., Dargent, P., Ferrari, M., Lemaitre, G.: Active optics concept for hypertelescope aberration control and pupil densification. In: Schultz, A.B. (ed.) High-Contrast Imaging for Exo-Planet Detection. SPIE Conf. Proc., 4860, pp. 371-380 (2003)

22. Kopeikin, S., Korobkov, P.: arXiv:gr-qc/0510084v1 (2005)

23. Ragazzoni, R., Valente, G., Marchetti, E.: Gravitational wave detection through microlensing. MNRAS 345, 100-110 (2003)

24. Le Coroller, H., Dejonghe, J., Arpesella, C., Vernet, D., Labeyrie, A.: Tests with a Carlinatype hypertelescope prototype. I. Demonstration of star tracking and fringe acquisition with a balloon-suspended focal camera. Astron. Astrophys. 426, 721-728 (2004)

25. Labeyrie, A., Le Coroller, H.: Extrasolar planet imaging. In: New Frontiers in Stellar Interferometry, Proc. SPIE 5491, 90 (2004)

26. Borkowski, V., Labeyrie, A., Martinache, F., Peterson, D.: Sensitivity of a "dispersedspeckles" piston sensor for multi-aperture interferometers and hypertelescopes. Astron. Astrophys. 429, 747-753 (2005)

27. Carpenter, K.G., Schrijver, C.J., Karovska, M.: The stellar imager (SI) vision mission. SPIE, May 24-31, \#6268-77 (2006) 
28. Paredes, J.M., Bosch-Ramon, V., Romero, G.E.: Astron. Astrophys. 451(1), 259-266 (2006)

29. Dubath, F., et al.: Astro-ph,/ 0603536v2 (2007)

30. Lardière, O., Martinache, F., Patru, F.: Direct imaging with highly diluted apertures - I. Fieldof-view limitations. MNRAS 375(3), 977-988 (2007)

31. Labeyrie, A.: Comparison of ELTs, interferometers and hypertelescopes for deep field imaging and coronagraphy. C. R. Physique 8 (2007)

32. Labeyrie, A., Le Coroller, H., Dejonghe, J.: Steps toward hypertelescopes on Earth and in space. SPIE Marseille (2008)

33. Labeyrie, A., et al.: Optical design solutions for hypertelescopes (2008, in preparation)

34. Labeyrie, A.: Extreme coronagraphy for detecting exo-Earths. In: Carbillet, M., Ferrari, A., Aime, C. (eds.) Astronomy with High Contrast Imaging III: Instrumental Techniques, Modeling and Data Processing. EAS Publications Series, vol. 22, pp. 189-198 (2006)

35. Labeyrie, A.: Standing waves and pellicle: a possible approach to very large space telescopes. Astron. Astrophys. 77, L1-L2 (1979)

36. Labeyrie, A., Fournier, J.M.R., Stachnik, R.V.: Laser-trapped mirrors in space: steps toward laboratory testing. In: Denver Proceedings of SPIE (2004)

37. Labeyrie, A.: Feasibility of a laser-driven hypertelescope in space (2008, in preparation)

38. Thompson, J.D., Zwickl, B.M., Jayich, A.M., Marquardt, F., Girvin, S.M., Harris, J.G.E.: Strong dispersive coupling of a high-finesse cavity to a micromechanical membrane. Nature $452(2008)$

39. Leizawitz, D.T., et al.: A SPECS update: engineering and technology requirements for a spacebased far-IR imaging interferometer. Proc. SPIE 5491, 212 (2004) 